\title{
La FMH, la plate-forme HIN et les bénéfices du numérique!
}

Urs Stoffel

Membre du Comité central de la $\mathrm{FMH}$, responsable du domaine eHealth - infrastructure de sécurité et président du Conseil d'administration de HIN

\section{Faire confiance à l'échange des données en ligne et à la communication électronique}

Alors que la mise en œuvre de la stratégie eHealth de la Confédération en est à ses débuts, la cybersanté suscite encore bien des réserves de la part des médecins et de la population, notamment face aux bénéfices et aux risques de cette nouvelle technologie. Nous avons donc pour but de dissiper ces craintes et de convaincre les patients et les médecins de l'utilité de la cybersanté.

\section{Nous devons proposer des solutions utiles et pragmatiques, susceptibles de répondre aux attentes de la base et des patients.}

Très tôt, la FMH a reconnu l'importance cruciale de la communication sécurisée dans le cadre des échanges électroniques. En fondant la plate-forme HIN il y a maintenant 16 ans, elle a posé un jalon capital pour consolider la confiance dans le réseau et assurer la confidentialité des échanges en ligne. Des milliers de médecins utilisent chaque jour les outils HIN avec succès.

\section{La plate-forme HIN étend son offre et investit dans des technologies d'avenir novatrices}

La FMH continuera à s'engager de manière ciblée dans le développement de la plateforme HIN pour qu'elle devienne un outil essentiel de l'utilisation sécurisée de la cybersanté. L'offre de prestations destinées aux membres de la «Communauté HIN» sera progressivement étendue afin de créer une véritable plus-value pour les abonnés. En accord avec la FMH, HIN développe des outils de communication novateurs qui facilitent le quotidien des médecins et leur permettent de travailler partout et en tout temps. D'une importance cruciale pour la FMH, la mise en réseau et l'échange des données doivent rester indépendants de services de transaction onéreux et reposer sur des institutions et des plate-formes propres au corps médical.

\section{Les bénéfices pour les patients et les méde- cins sont au cœur de nos préoccupations}

Nous disposons d'ores et déjà d'exemples d'utilisation réussie de la cybersanté: la vérification médicale de l'aptitude à la conduite des offices de la circulation s'effectue en ligne; l'envoi sécurisé des rapports de sortie d'hôpital et un accès simple et sécurisé aux dossiers médicaux à l'hôpital, comme à l'Hôpital de Zurich, sont des applications pratiques

\section{La plate-forme HIN actuelle sera développée pour devenir un outil essentiel de l'utilisation sécurisée de la cybersanté.}

La plate-forme HIN assure la protection des données et préserve le secret médical dans la communication électronique. En outre, elle vérifie et authentifie l'identité des utilisateurs avant chaque utilisation, ce qui inspire encore plus de confiance en ces nouvelles technologies.

Mais le fossé entre la vision idéale de ce que devrait être la cybersanté et la réalité est encore profond. C'est pour cette raison que nous devons proposer des solutions utiles et pragmatiques, susceptibles de répondre aux attentes de la base et des patients. Nous devons également porter un regard attentif sur ce qui se fait dans les pays voisins dans ce domaine. En apprenant de leurs erreurs, nous pourrons y remédier, là où c'est nécessaire. de la cybersanté. Et nous avons aujourd'hui également la possibilité de communiquer de manière sécurisée avec nos patients par courriel (Secure Mail GLOBAL). Toutes ces solutions ont été développées sur la base de la plate-forme HIN.

Néanmoins, il reste encore beaucoup à faire... La FMH souhaite relever ces défis à venir en collaboration avec HIN. Elle veut soutenir et encourager le développement d'outils appropriés à une mise en œuvre de la cybersanté bénéfique aux patients et aux médecins en veillant à ce que ces outils soient adaptés aux enjeux du numérique.
FMH

Elfenstrasse 18

CH-3000 Berne 15

Tél. 0313591111

Fax 0313591112 